\section{RSP}

http://www.rsp.fsp.usp.br/
Revista de Saúde Pública

\title{
Notificação da infecção pelo HIV em gestantes: estimativas a partir de um estudo nacional
}

\author{
Rosa Maria Soares Madeira Domingues', Valéria Saraceni", Maria do Carmo Leal'"' \\ ' Fundação Oswaldo Cruz. Instituto Nacional de Infectologia Evandro Chagas. Laboratório de Pesquisa Clínica \\ em DST/Aids. Rio de Janeiro, RJ, Brasil \\ " Secretaria Municipal de Saúde do Rio de Janeiro. Superintendência de Vigilância em Saúde. Coordenação de \\ Análise da Situação de Saúde. Rio de Janeiro, RJ, Brasil \\ III Fundação Oswaldo Cruz. Escola Nacional de Saúde Pública Sérgio Arouca. Departamento de Epidemiologia e \\ Métodos Quantitativos em Saúde. Rio de Janeiro, RJ, Brasil
}

Correspondência:

Rosa Maria Soares Madeira Domingues

Instituto Nacional de Infectologia

Evandro Chagas - Fiocruz

Laboratório de Pesquisa Clínica em

DST/Aids

Avenida Brasil, 4365 Manguinhos 21040-360 Rio de Janeiro, RJ, Brasil

E-mail: rosa.domingues@ini.fiocruz.br

Recebido: 23 ago 2017

Aprovado: 10 out 2017

Como citar: Domingues RMSM, Saraceni V, Leal MC. Notificação da infecção pelo HIV em gestantes: estimativas a partir de um estudo nacional. Rev Saude Publica. 2018;52:43

Copyright: Este é um artigo de acesso aberto distribuído sob os termos da Licença de Atribuição Creative Commons, que permite uso irrestrito, distribuição e reprodução em qualquer meio, desde que o autor e a fonte originais sejam creditados.

\section{RESUMO}

OBJETIVO: Estimar a cobertura de notificação de casos de infecção pelo HIV em gestantes, o aumento na cobertura de notificação a ser obtido pela busca rotineira de dados em outros sistemas de informação nacionais, e identificar oportunidades perdidas de identificação de gestantes HIV+ em maternidades brasileiras.

MÉTODOS: Estudo descritivo, de relacionamento de base de dados de estudo nacional com coleta primária de dados (estudo "Nascer no Brasil"), e de base de dados secundários de sistemas de informação nacionais. O estudo "Nascer no Brasil" é um estudo de base nacional, realizado em 2011-2012, com 23.894 puérperas, que identificou gestantes infectadas pelo HIV, utilizando registros em cartão de pré-natal e prontuário. Casos de gestantes com infecção pelo HIV identificadas no estudo "Nascer no Brasil" foram pesquisadas no Sistema de Informação de Agravos de Notificação, Sistema de Controle de Exames Laboratoriais da Rede Nacional de Contagem de Linfócitos CD4+/CD8+ e Carga Viral, e Sistema de Controle Logístico de Medicamentos. Utilizou-se o software OpenRecLink para relacionamento das bases de dados. Foi estimada a cobertura de notificação, com respectivo intervalo de confiança, dos sistemas de informação nacionais avaliados.

RESULTADOS: A cobertura de notificação de HIV em gestantes no Sistema de Informação de Agravos de Notificação foi estimada em 57,1\% (IC95\% 42,9-70,2), e 89,3\% das gestantes HIV+ (IC95\% 81,2-94,2) foram localizadas em algum dos sistemas de informação nacionais pesquisados. A busca em outros sistemas de informação nacionais resultaria em aumento de $57,1 \%$ dos casos notificados. Não foram identificadas oportunidades perdidas de diagnóstico de gestantes HIV+ nas maternidades avaliadas pelo estudo "Nascer no Brasil".

CONCLUSÕES: A busca rotineira de informação em outros sistemas de informação nacionais, procedimento utilizado pelo Ministério da Saúde para casos de Aids em adultos e em crianças, deve ser adotado para os casos de infecção pelo HIV na gestação.

DESCRITORES: Gestantes. Infecções por HIV, diagnóstico. Cuidado Pré-Natal. Sistemas de Informação em Saúde. Serviços de Vigilância Epidemiológica. 


\section{INTRODUÇÃO}

A infecção pelo HIV na gestação é um agravo de notificação compulsória no Brasil desde 2006. A taxa de detecção de casos de infecção pelo HIV em gestantes no país apresenta tendência de aumento nos últimos 10 anos, alcançando o valor de 2,7 por 1.000 nascidos vivos em 2015. Diferenças nas taxas de detecção são observadas nas diversas regiões. As regiões Norte e Nordeste apresentaram os maiores incrementos dessa taxa de 2006 a 2015, enquanto a região Sul apresentou a maior taxa de detecção em todo o período, com valor 2,2 vezes maior que a do país em $2015^{1}$.

Apesar de melhorias na notificação desses casos, estima-se que a subnotificação ainda seja elevada. Estudos Sentinela-Parturiente realizados em $2006^{2}$ e 2010/2012 em hospitas públicos estimaram uma prevalência de infecção pelo HIV em gestantes no país de 0,41\% e $0,38 \%$, respectivamente. Considerando-se uma estimativa de cerca de 11.070 casos anuais (calculada a partir da prevalência de 0,38\% de infecção pelo HIV em gestantes, medida em 2010/2012) e a notificação de 7.901 casos em 2015, pode-se estimar que aproximadamente $70,0 \%$ dos casos de infecção pelo HIV na gestação foram notificados. Isso indica dificuldades no diagnóstico ou na notificação dos casos.

Dados de quatro sistemas de informação - Sistema de Informação de Agravos de Notificação (SINAN), Sistema da Informação de Mortalidade (SIM), Sistema de Controle de Exames Laboratoriais da Rede Nacional de Contagem de Linfócitos CD4+/CD8+ e Carga Viral do HIV (SISCEL) e Sistema de Controle Logístico de Medicamentos (SICLOM) - são utilizados rotineiramente como fonte para obtenção de dados pelo Ministério da Saúde, para aumentar e qualificar os dados de notificação de Aids em crianças e adultos. Considerando a série histórica de 2000 a 2016, do total de 634.051 casos de Aids registrados, somente 70,3\% foram oriundos do SINAN, ressaltando a importância dessa estratégia para melhor estimativa dos casos. Entretanto, essa rotina não está implantada para os casos de infecção pelo HIV em gestantes.

O estudo "Nascer no Brasil" é um estudo nacional de base hospitalar, realizado em 2011-2012, que permitiu a identificação de gestantes infectadas pelo HIV a partir de dados registrados no cartão de pré-natal e prontuários hospitalares. Considerando-se as falhas no sistema de notificação de casos de infecção pelo HIV em gestantes, este estudo teve por objetivo estimar a cobertura de notificação de casos de infecção pelo HIV em gestantes no SINAN, estimar o aumento na cobertura de notificação a ser obtido pela busca rotineira de dados em outros sistemas de informação (SIM, SISCEL, SICLOM) e identificar oportunidades perdidas de prevenção da transmissão vertical em maternidades brasileiras tomando por referência os resultados do estudo "Nascer no Brasil".

\section{MÉTODOS}

Estudo descritivo de relacionamento de base de dados com coleta primária de dados (estudo "Nascer no Brasil"), e de base de dados secundários de Sistemas de Informação Nacionais (SINAN, SIM, SISCEL, SICLOM).

A pesquisa "Nascer no Brasil", estudo nacional de base hospitalar, foi realizada entre fevereiro de 2011 e outubro de 2012. Teve por objetivo avaliar a assistência pré-natal, ao parto e aos recém-natos, e os resultados dessa assistência. Foram incluídas no estudo 23.894 mulheres internadas em 266 hospitais públicos e privados em todas as regiões do país. Todas as puérperas com nascidos vivos no hospital em que o feto tinha qualquer idade gestacional ou peso, ou um natimorto em que o feto tinha idade gestacional superior a 22 semanas ou peso superior a 500 gramas, foram consideradas elegíveis para o estudo. Maiores informações sobre o desenho do estudo e do cálculo amostral podem ser obtidos em Leal ${ }^{4} \mathrm{eV}$ Vasconcellos ${ }^{5}$.

Como base de dados secundários, foram utilizados os Sistemas de Informação Nacionais: SINAN, SISCEL e SICLOM. Planejou-se utilizar também o SIM, mas não foi possível obter essa base de dados. 
Casos de "gestantes infectadas pelo HIV", de "crianças expostas ao HIV" e de "Aids" são agravos de notificação compulsória no país. Modelos específicos de fichas de notificação para cada um desses agravos são utilizados nacionalmente pelo SINAN. Os casos notificados são processados pelas Secretarias Municipais e Estaduais de Saúde, e os dados são enviados ao Ministério da Saúde, que disponibiliza os dados nacionais referentes ao ano de diagnóstico do agravo.

O SICLOM tem o objetivo de gerenciar a logística dos medicamentos antirretrovirais (ARV) no Brasil. Todos os medicamentos antirretrovirais fornecidos a gestantes infectadas pelo HIV, a parturientes, a crianças expostas ao HIV e a pessoas (adultos e crianças) com Aids são registrados nesse sistema.

O Sistema de Controle de Exames Laboratoriais de CD4/CD8 e Carga Viral é utilizado no país desde 1997 para o controle de exames de contagem de CD4/CD8 e carga viral em pessoas vivendo com HIV e Aids. O SISCEL é utilizado em todos os estados do Brasil e todas as informações são armazenadas no banco de dados central, que fica no Departamento de DST, Aids e Hepatites Virais.

Para o primeiro objetivo, "estimar a cobertura de notificação de casos de infecção pelo HIV em gestantes", foi realizada uma busca nominal dos casos de gestantes com diagnóstico de infecção pelo HIV identificadas no estudo "Nascer no Brasil" e no SINAN.

Para identificação dos casos de Gestantes infectadas pelo HIV no estudo "Nascer no Brasil", foram utilizados dados de cartão de pré-natal e de prontuário hospitalar, segundo os seguintes critérios $^{6}$ a) resultados reagentes de sorologia para o HIV registrados no cartão de pré-natal (dois testes rápidos ou Elisa + imunofluorescência ou Elisa + Western Blot); ou b) registros nos arquivos médicos hospitalares de qualquer uma das seguintes situações: diagnóstico de infecção por HIV; indicação de cesariana devido à infecção pelo HIV; utilização de AZT durante o trabalho de parto ou parto; uso de xarope de AZT pelo recém-nascido; suspensão da amamentação devido à infecção materna pelo HIV; registro do diagnóstico de "criança exposta ao HIV".

Para identificação das gestantes infectadas pelo HIV no SINAN, foram selecionados casos de "HIV na gestação" notificados ao SINAN de 1/5/2010 a 31/10/2012. A coleta de dados do estudo "Nascer no Brasil" ocorreu de fevereiro/2011 a outubro/2012. A inclusão de 2010 visou identificar gestantes com parto a partir de fevereiro de 2011 que tivessem sido notificadas ao SINAN durante a gestação em 2010.

Para o segundo objetivo, "estimar o aumento na cobertura de notificação a ser obtido pela busca rotineira de dados em outros sistemas de informação", foram utilizados dados nos sistemas SINAN, SICLOM e SISCEL. No SINAN, foram pesquisados casos de Aids em adultos. Essa busca visou identificar mulheres previamente notificadas com Aids que não tivessem sido notificadas como gestantes infectadas pelo HIV durante a gestação. Para essa busca foram utilizados dados do SINAN até 31/10/2012, data de encerramento da coleta de dados da pesquisa "Nascer no Brasil”. Planejou-se a procura de casos de crianças expostas ao HIV, visando identificar crianças expostas durante a gestação e o parto, cuja mãe não tivesse sido notificada. Entretanto, essa ficha de investigação não se encontra implantada e não há uma base de dados disponível para consulta.

Todos os casos registrados no SICLOM ou no SISCEL de gestantes infectadas, crianças expostas ao HIV ou crianças infectadas pelo HIV/Aids foram selecionadas neste estudo, por se tratar de sistemas específicos para portadores da infecção pelo HIV. Para as gestantes infectadas, foram considerados registros feitos de 1/5/2010 a 31/10/2012, visando identificar gestantes com parto em 2011 que possam ter sido registradas no SISCEL ou no SICLOM durante a gestação em 2010. Para as crianças expostas ao HIV, foram considerados registros feitos de 1/2/2011 a 31/12/2012, visando identificar crianças nascidas em outubro de 2012 com uso de medicação antirretroviral por seis semanas após o parto. Para crianças infectadas pelo HIV, foi utilizado o período de 1/2/2011 a 31/6/2016.

Os casos identificados nos sistemas foram comparados aos casos notificados como infecção pelo HIV na gestação no SINAN identificados no primeiro objetivo. 
Para o terceiro objetivo, "identificar oportunidades perdidas de prevenção da transmissão vertical em maternidades brasileiras”, todas as puérperas sem diagnóstico de infecção pelo HIV no estudo "Nascer no Brasil" foram relacionadas aos Sistemas de Informação Nacionais SINAN, SISCEL e SICLOM. Casos de infecção pelo HIV na gestação e Aids em adultos notificadas ao SINAN e gestantes registradas no SISCEL e SICLOM foram considerados não identificados pelas maternidades, representando perda de oportunidade de prevenção da transmissão vertical durante a assistência ao parto. Os mesmos períodos de busca em cada sistema de informação descritos no segundo objetivo foram utilizados para o terceiro objetivo.

Os seguintes procedimentos foram utilizados para o relacionamento das bases de dados: 1) Limpeza e padronização das variáveis constando das bases do estudo "Nascer no Brasil"; SINAN Aids adulto previamente relacionado com o SIM; SINAN Gestante HIV-positiva; Cadastro no SICLOM e SISCEL utilizando o Stata 11.0;2) Relacionamento (linkage) das bases de dados usando o software OpenRecLink (ORL). Ordem dos relacionamentos entre as bases de dados: Base Nascer e Cadastro no SICLOM e SISCEL; Base Nascer e SINAN Gestante HIVpositiva; Base Nascer e SINAN Aids adulto.

Os seguintes procedimentos foram utilizados em todos os relacionamentos:

- Variáveis para relacionamento: nome da parturiente, nome da mãe, data de nascimento, cidade de residência (esta para inspeção visual).

- Blocagem:

Passo 1: PBLOCO (1º nome) + UBLOCO (último nome) + ANONASC (ano de nascimento)

Passo 2: PBLOCO + ANONASC + PBLOCO_mae (1º nome da mãe)

Passo 3: PBLOCO + UBLOCO + PBLOCO_mae

- Parâmetros das variáveis para relacionamento:

Nome: Tipo “Aproximado”, Correto “92”, Incorreto “1” e Limiar “85”

Nome da mãe: Tipo “Aproximado”, Correto “92”, Incorreto “1” e Limiar “85”

Data de nascimento: Tipo “Caractere", Correto “90”, Incorreto “5” e Limiar “65”, de acordo com o Manual do Reclink III? .

Os endereços das mulheres encontradas sofreram inspeção visual como conferência. Devido ao pequeno número de pares encontrados, as mulheres não localizadas foram procuradas nominalmente nas bases do SINAN e SICLOM/SISCEL. Após o relacionamento, foi construída uma base final em que constavam os casos encontrados nos vários sistemas e as mulheres incluídas no estudo "Nascer no Brasil".

Para o cálculo da cobertura de notificação de gestantes com infecção pelo HIV no SINAN, foi estimada a proporção de gestantes com diagnóstico de infecção pelo HIV identificadas pelo estudo "Nascer no Brasil” (NB), notificadas ao SINAN no país e por macrorregião, com intervalo de $95 \%$ de confiança.

A descrição dos casos identificados em cada Sistema de Informação (SI) foi feita para avaliar o aumento da cobertura de notificação que seria obtido com o uso rotineiro de outras fontes de informação além do SINAN (SISCEL, SICLOM e SIM). Além disso, foi estimado o aumento da cobertura que seria obtido com o uso rotineiro dessas fontes de informação.

A proporção de gestantes com diagnóstico de infecção pelo HIV (identificadas pelo estudo "Nascer no Brasil" e pelos SIS) que não foram identificadas pelas maternidades que participaram do estudo "Nascer no Brasil” foi verificada para o cálculo das oportunidades perdidas de prevenção da transmissão vertical. 
O estudo "Nascer no Brasil" utilizou um desenho amostral complexo que foi considerado em todas as análises estatísticas com utilização de procedimentos de ponderação e calibração ${ }^{6}$ para todos os dados (números e proporções) apresentados neste estudo. As análises foram realizadas com o programa estatístico IBM SPSS Statistics para Windows, versão 19.0 (IBM Corp., Armonk, NY, EUA).

O presente estudo foi aprovado pelo Comitê de Ética em Pesquisa da Escola Nacional de Saúde Pública Sérgio Arouca da Fundação Oswaldo Cruz (Parecer 1.647.494/2016). As bases nominais dos sistemas descritos foram disponibilizadas pelo Ministério da Saúde por meio da assinatura de um termo de responsabilidade. Não foi utilizado termo de consentimento livre e esclarecido por se tratar de análise de bases de dados. Apenas os membros da equipe tiveram acesso às bases nominais cedidas pelo Ministério da Saúde e todos os cuidados foram adotados visando garantir o sigilo e a confidencialidade das informações.

\section{RESULTADOS}

Do total de casos de gestantes infectadas pelo HIV relatadas no estudo "Nascer no Brasil" ( $\mathrm{n}=74), 89,3 \%$ foram identificados em algum sistema de informação: 73,1\% no SICLOM; $60,4 \%$ no SISCEL Carga Viral; 59,3\% no SISCEL CD4; 57,1\% no SINAN Gestante HIV; $42,6 \%$ no SINAN Aids e 3,7\% no SIM (Tabela 1, Figura 1). A maioria das mulheres foi identificada em mais de um sistema, com uma pequena proporção reconhecida em apenas um dos sistemas de informação: 3,9\% no SICLOM, 5,3\% no SINAN Gestante HIV, e 1,1\% no SINAN Aids.

A identificação de casos de gestantes com infecção pelo HIV em algum sistema variou de $75,3 \%$ na região Norte a 96,7\% na região Sul. Variações regionais maiores foram observadas para a notificação de gestantes com infecção pelo HIV no SINAN. A menor cobertura foi observada na região Norte $(20,2 \%)$ e a maior na região Nordeste $(79,5 \%)$ (Tabela 2).

Considerando os casos identificados em algum dos Sistemas de Informação, a prevalência de infecção pelo HIV na gestação foi estimada em 0,3\% (IC95\% 0,21-0,36). Utilizando-se apenas os casos notificados ao SINAN gestantes HIV+, a prevalência estimada de infecção pelo HIV na gestação foi de 0,2\% (IC95\% 0,13-0,23). Com a busca rotineira de casos em outros sistemas de informação, seria possível aumentar o número de casos no SINAN Gestante HIV em 57,1\%. O maior aumento seria obtido com a busca no SICLOM, que representaria um acréscimo de 52,4\% nos casos notificados, seguido do SISCEL CD4 e CV (42,9\%) e SINAN Aids (16,7\%) (Tabela 3, Figura 2).

Dos casos de gestantes com HIV identificados nos Sistemas de Informação, seis não foram diagnosticados pelas maternidades que participaram do estudo "Nascer no Brasil". Dos seis casos identificados, cinco apresentaram diagnóstico da infecção pelo HIV em gestação posterior à avaliada pelo estudo "Nascer no Brasil", e uma gestante teve diagnóstico em

Tabela 1. Proporção de casos de gestantes infectadas pelo HIV identificadas no estudo "Nascer no Brasil", localizadas nos sistemas de informação SINAN, SICLOM e SISCEL. Brasil, 2011 a 2012.

\begin{tabular}{lcc}
\hline Sistema de informação (SIS) & $\%$ & IC95\% \\
\hline SICLOM & 73,1 & $61,9-82,0$ \\
SISCEL Carga Viral & 60,4 & $48,1-71,6$ \\
SISCEL CD4 & 59,3 & $46,9-70,6$ \\
SINAN Gestante HIV+ & 57,1 & $42,9-70,2$ \\
SINAN Aids & 42,6 & $31,6-54,5$ \\
SIM & 3,7 & $1,0-12,0$ \\
Algum Sistema de Informação em Saúde & 89,3 & $81,2-94,2$ \\
\hline
\end{tabular}

SINAN: Sistema de Informação de Agravos de Notificação; SISCEL: Sistema de Controle de Exames Laboratoriais da Rede Nacional de Contagem de Linfócitos CD4+/CD8+ e Carga Viral do HIV; SICLOM: Sistema de Controle Logístico de Medicamentos; SIM: Sistema da Informação de Mortalidade 


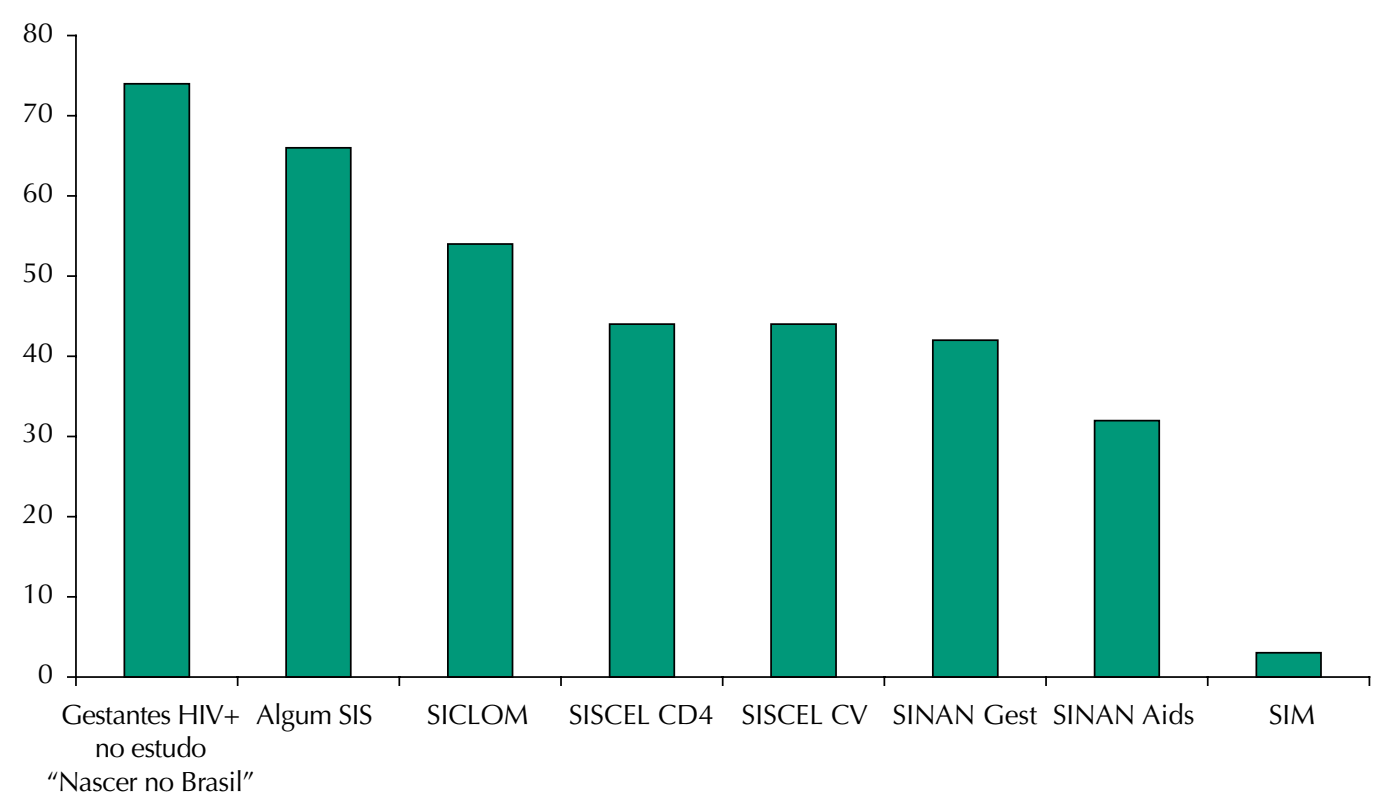

SIS: Sistema de Informação em Saúde; SINAN Gest: Sistema de Informação de Agravos de Notificação Gestante HIV+; SINAN Aids: Sistema de Informação de Agravos de Notificação Aids; SIM: Sistema da Informação de Mortalidade; SISCEL CD4: Sistema de Controle de Exames Laboratoriais da Rede Nacional de Contagem de Linfócitos CD4+/CD8+; SISCEL CV: Sistema de Controle de Exames Laboratoriais da Rede Nacional de Carga Viral do HIV; SICLOM: Sistema de Controle Logístico de Medicamentos

Figura 1. Número de gestantes com infecção pelo HIV identificadas no estudo "Nascer no Brasil" e nos Sistemas de Informação Nacionais. Brasil, 2011 a 2012.

Tabela 2. Proporção de gestantes com infecção pelo HIV identificadas no estudo "Nascer no Brasil", segundo localização em algum dos Sistemas de Informação Nacionais (SINAN Aids, SICLOM, SISCEL) e no sistema SINÁN "Gestante HIV+" por macrorregião do país. Brasil, 2011 a 2012.

\begin{tabular}{lccccc}
\hline \multirow{2}{*}{ Região } & \multicolumn{2}{c}{ Algum Sistema de Informação } & & \multicolumn{2}{c}{ SINAN Gestante HIV+ } \\
\cline { 2 - 3 } \cline { 5 - 6 } & $\%$ & IC95\% & & $\%$ & IC95\% \\
\hline Norte & 75,3 & $62,7-84,7$ & & 20,2 & $2,3-73,1$ \\
Nordeste & 84,5 & $79,6-88,4$ & & 79,5 & $73,8-84,3$ \\
Centro-Oeste & 76,3 & $66,6-83,9$ & & 76,3 & $66,6-83,9$ \\
Sudeste & 92,1 & $55,9-99,1$ & & 54,9 & $25,4-81,3$ \\
Sul & 96,7 & $77,1-99,6$ & & 57,0 & $42,0-70,8$ \\
\hline Total & 89,3 & $81,2-94,2$ & & 57,1 & $42,9-70,2$ \\
\hline
\end{tabular}

SINAN Gestante HIV+: Sistema de Informação de Agravos de Notificação Gestante HIV+; SICLOM: Sistema de Controle Logístico de Medicamentos; SISCEL: Sistema de Controle de Exames Laboratoriais da Rede Nacional de Contagem de Linfócitos CD4+/CD8+ e Carga Viral do HIV

Tabela 3. Proporção de gestantes HIV+ identificadas no estudo "Nascer no Brasil", segundo notificação no SINAN "gestante HIV+" e identificação em outros Sistemas de Informação. Brasil, 2011 a 2012.

\begin{tabular}{lccc}
\hline & \multicolumn{2}{c}{ SINAN gestante HIV } & \\
\cline { 2 - 3 } Sistema de Informação (SIS) & Não (32) & Total \\
\cline { 2 - 3 } \cline { 2 - 2 } & $\mathbf{n}(\%)$ & $\mathbf{n}(\%)$ & \\
\hline SICLOM & $22(70,9)$ & $31(74,7)$ & 53 \\
SISCEL CV & $18(57,4)$ & $26(62,7)$ & 44 \\
SISCEL CD4 & $18(57,4)$ & $25(60,7)$ & 43 \\
SINAN Aids & $7(21,7)$ & $25(58,4)$ & 32 \\
SIM & 0 & $3(100)$ & 3 \\
Algum SIS & $24(75,1)$ & $42(100)$ & 66 \\
\hline
\end{tabular}

SINAN: Sistema de Informação de Agravos de Notificação; SIM: Sistema da Informação de Mortalidade; SISCEL: Sistema de Controle de Exames Laboratoriais da Rede Nacional de Contagem de Linfócitos CD4+/CD8+ e Carga Viral do HIV; SICLOM: Sistema de Controle Logístico de Medicamentos 


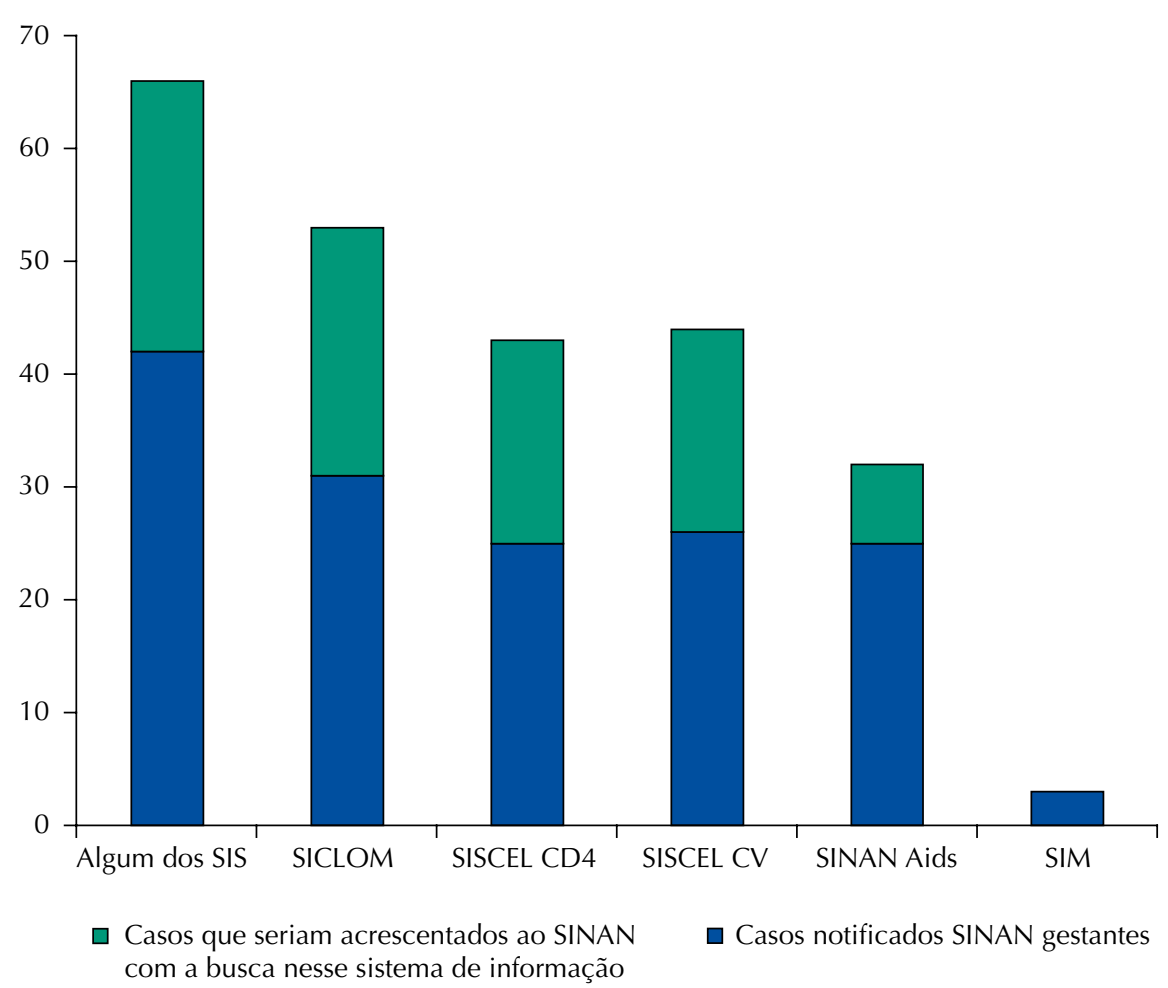

SIS: Sistema de Informação em Saúde; SINAN Aids: Sistema de Informação de Agravos de Notificação Aids; SIM: Sistema da Informação de Mortalidade; SISCEL CD4: Sistema de Controle de Exames Laboratoriais da Rede Nacional de Contagem de Linfócitos CD4+/CD8+; SISCEL CV: Sistema de Controle de Exames Laboratoriais da Rede Nacional de Carga Viral do HIV; SICLOM: Sistema de Controle Logístico de Medicamentos

Figura 2. Casos notificados ao SINAN HIV na gestação e casos que seriam acrescentados com a busca em outros Sistemas de Informação nacionais. Brasil, 2011 a 2012.

gestação anterior, apresentando sorologia "não reagente" para o HIV na gestação atual, o que representa um provável diagnóstico falso-positivo na gestação anterior.

\section{DISCUSSÃO}

O resultado obtido, de notificação de 57,1\% das gestantes no estudo "Nascer no Brasil" no SINAN HIV na gestação, é um pouco inferior à estimativa de cobertura de notificação calculada a partir dos dados de prevalência dos estudos sentinela parturientes (aproximadamente $70,0 \%)$. Devido ao pequeno número de casos identificados, a estimativa obtida é pouco precisa, com intervalo de confiança amplo e, por isso, deve ser analisada com cautela, principalmente para as macrorregiões do país.

Estudos anteriores avaliaram a notificação de casos de gestantes infectadas pelo HIV no Brasil. Lemos et al., utilizando técnica de captura e recaptura com os dados do SINAN, SISCEL e de prontuário clínico em Sergipe estimaram uma subnotificação de $34,3 \%^{8}$. A subnotificação foi menor em Vitória de 2000 a 2006, atingindo 4,9\% entre as gestantes portadoras de HIV ${ }^{9}$.

A identificação de casos de gestantes infectadas pelo HIV em outros Sistemas de Informação, principalmente no SICLOM e no SISCEL, seguiu o mesmo padrão observado na rotina utilizada pelo Ministério da Saúde para identificação de casos de Aids em adultos e crianças. Segundo o último boletim epidemiológico do Ministério da Saúde ${ }^{1}$, de 39.113 casos registrados de Aids em 2015, 56,3\% eram do SINAN, 7,4\% do SIM e 36,3\% do SISCEL/SICLOM.

Avaliando os dados das gestantes e crianças portadoras de HIV em cinco estados e no Distrito Federal, Miranda et al. mostraram que a melhor fonte para encontrar crianças menores de 13 anos portadoras do HIV foi o SISCEL; o SINAN continha menos de um terço dos $\operatorname{casos}^{10}$. 
Segundo resultados do presente estudo, a busca nos SIS permitiria ampliar a notificação de casos de HIV na gestação em 57,0\% e o sistema que mais resultaria na identificação de novos casos seria o SICLOM. Segundo o Ministério da Saúde ${ }^{1}$, uma nova funcionalidade no SICLOM foi desenvolvida com o objetivo de informar os pacientes portadores de HIV/Aids que ainda não foram notificados no SINAN. Essa funcionalidade poderia ser utilizada para os casos de HIV na gestação, identificando mulheres não notificadas na gestação durante o fornecimento da medicação antirretroviral.

Uma possibilidade não investigada neste estudo seria a busca de gestantes no Sistema de Informações Hospitalares (SIH) internadas por abortamento. Ainda que restrito a serviços públicos, o SIH seria o único que permitiria a identificação de gestantes infectadas pelo HIV, cujo desfecho da gestação tenha sido um abortamento. Um estudo realizado no Rio de Janeiro, RJ, que tinha por objetivo estimar a incidência de sífilis congênita em gestantes acompanhadas na rede SUS ${ }^{11}$, realizou a busca de casos no SIH, o que resultou na identificação de duas gestantes infectadas pela doença que não haviam sido notificadas ao SINAN. Além de aumentar o número de casos de sífilis congênita e de sífilis na gestação, essa busca permitiu identificar um desfecho negativo da infecção pela sífilis na gestação (abortamento) que teria sido perdido pelos sistemas de informação. Entretanto, os dados deste estudo não permitem estimar o tipo de resultado que seria obtido no caso da infecção pelo HIV.

Neste estudo, as gestações ocorridas no período foram identificadas na base de dados do estudo "Nascer no Brasil". O Sistema de Informações sobre Nascidos Vivos (SINASC) - sistema com cobertura universal, que abrange nascimentos em hospitais públicos, privados e domicílios - seria o sistema a ser utilizado para identificação de gestações que resultaram em nascimentos vivos. Já o SIM, também com cobertura universal, ainda que com variações regionais, seria o sistema a ser utilizado para a identificação de gestações que resultaram em óbitos fetais. Embora o SIH apresente cobertura apenas de internações ocorridas no setor público, poderia ser utilizado para a identificação de gestações que resultaram em abortamento.

Todos os casos de gestantes com HIV identificados nos Sistemas de Informação foram localizados pelas maternidades que participaram do estudo "Nascer no Brasil", já que os casos não diagnosticados ocorreram em gestação posterior à avaliada pelo estudo ou não se confirmaram na gestação atual. Esse resultado sugere que mecanismos de identificação de gestantes infectadas pelo HIV têm sido utilizados em maternidades de maior porte, com mais de 500 partos/ano, e a subnotificação identificada é decorrente de falhas no preenchimento ou envio das fichas de notificação. Como a testagem sorológica para o HIV durante o pré-natal no país não atingiu a cobertura de $100 \%$, mecanismos de diagnóstico na admissão para o parto, como o uso de testes rápidos devem estar sendo utilizados e precisam continuar a ser incentivados para evitar oportunidades perdidas do diagnóstico da infecção. Como o estudo "Nascer no Brasil" não incluiu maternidades com menos de 500 partos/ano, nem partos no domicílio ou em via pública, as oportunidades perdidas do diagnóstico da infecção pelo HIV nesses contextos devem ser investigadas em estudos futuros.

Este estudo apresenta algumas limitações. Foram incluídas no estudo "Nascer no Brasil" apenas puérperas internadas em hospitais com mais de 500 partos/ano e excluídas mulheres com internação por abortamento. Portanto, as mulheres identificadas como gestantes infectadas pelo HIV neste estudo não representam o universo de gestantes brasileiras. Não é possível avaliar a cobertura do SINAN gestante para esses casos. O estudo também não realizou os testes sorológicos para o HIV, e a identificação dos casos foi baseada em dados secundários, provenientes de registros em cartões de pré-natal e prontuários. É possível que tenha havido erros na identificação das gestantes, principalmente se mulheres que realizaram apenas um teste rápido na admissão para o parto foram classificadas como infectadas pelo HIV, sem confirmação posterior. Por último, não foi possível analisar a base de dados do SIM, o que impediu o relacionamento com esse sistema de informação. Entretanto, o SIM, bem como o SISCEL e o SICLOM, são relacionados rotineiramente ao SINAN. A busca nominal no SISCEL e no SICLOM não resultou em casos adicionais aos identificados na base SINAN. 
Assim, acreditamos que a ausência de relacionamento com o SIM não tenha acarretado perda de casos.

Este estudo identificou subnotificação expressiva de casos de gestantes infectadas pelo HIV no SINAN. A busca de casos em outros Sistemas de Informação, rotina já adotada pelo Departamento Nacional de DST, Aids e Hepatites virais para casos de Aids em adultos e crianças, permitiria ampliar a notificação de casos de HIV na gestação. Essa medida seria complementar ao incentivo ao aumento da notificação de casos de HIV na gestação, já que diversas informações relevantes para a vigilância desse agravo estão disponíveis apenas no SINAN.

\section{REFERÊNCIAS}

1. Ministério da Saúde (BR), Secretaria de Vigilância em Saúde, Departamento de DST, Aids e Hepatites Virais. Bol Epidemiol HIV/Aids. Brasília(DF); 2016 [citado 14 ago 2017]. Disponível em: http://www.aids.gov.br/pt-br/pub/2016/boletim-epidemiologico-de-aids-2016

2. Szwarcwald CL, Barbosa Junior A, Miranda AE, Paz LC. Resultados do Estudo SentinelaParturiente, 2006: desafios para o controle da sífilis congênita no Brasil. DST J Bras Doenças Sex Transm. 2007 [citado 14 ago 2017];19(3-4):128-33. Disponível em: http://www.dst.uff.br// revista19-3-2007/3.pdf

3. Pereira GF, Sabidó M, Caruso A, Oliveira SB, Mesquita F, Benzaken AS. HIV Prevalence among Pregnant Women in Brazil: a national survey. Rev Bras Ginecol Obstet. 2016;38(8):391-8. https://doi.org/10.1055/s-0036-1592102

4. Leal MC, Silva AAM, Dias MAB, Gama SGN, Rattner D, Moreira ME, et al. Birth in Brazil: national survey into labour and birth. Reprod Health. 2012;9:15. https://doi.org/10.1186/1742-4755-9-15

5. Vasconcelos MTL, Silva PLN, Pereira APE, Schilithz AOC, Souza Junior PRB, Szwarcwald CL. Desenho da amostra Nascer no Brasil: Pesquisa Nacional sobre Parto e Nascimento. Cad Saude Publica. 2014;30 Supl 1:S49-58. https://doi.org/10.1590/0102-311X00176013

6. Domingues RM, Szwarcwald CL, Souza Jr PR, Leal MC. Prenatal testing and prevalence of HIV infection during pregnancy: data from the "Birth in Brazil" study, a national hospital-based study. BMC Infect Dis. 2015;15:100. https://doi.org/10.1186/s12879-015-0837-8

7. Camargo Jr KR, Coeli CM. Reclink: aplicativo para o relacionamento de bases de dados, implementando o método probabilistic record linkage. Cad Saude Publica. 2000;16(2):439-47. https:/doi.org/10.1590/S0102-311X2000000200014

8. Lemos LMD, Duarte GS, Martins NGR, Silva FJCP, Ilozue C, Gurgel RQ. Estimating the number of HIV-positive pregnant women in Sergipe, Brazil, using capture-recapture. AIDS Care. 2013;25(6):691-4. https://doi.org/10.1080/09540121.2013.764389

9. Cerqueira ACB, Miranda AEB, Maciel ELN. Completude do banco de dados de gestante HIV positivo e de Aids em menores de treze anos do Sistema de Informação de Agravos de Notificação: Vitória, 2000 a 2006. Cad Saude Coletiva. 2010 [citado 14 ago 2017];18(1):191-4. Disponível em: http://www.cadernos.iesc.ufrj.br/cadernos/images/csc/2010_1/artigos/ Modelo\%20Livro\%20UFRJ\%2019-a.pdf

10. Miranda AE, Pereira GFM, Araujo MAL, Silveira MF, Tavares LL, Silva LCF, et al. Avaliação da cascata de cuidado na prevenção da transmissão vertical do HIV no Brasil. Cad Saude Publica. 2016;32(9):e00118215. https://doi.org/10.1590/0102-311X00118215

11. Domingues RMSM, Saraceni V, Hartz ZMA, Leal MC. Congenital syphilis: a sentinel event in antenatal care quality. Rev Saude Publica. 2013;47(1):147-57. https://doi.org/10.1590/S0034-89102013000100019

Contribuição dos Autores: Concepção e planejamento do estudo, coleta dos dados, análise e interpretação dos dados: RMSMD, VS. Elaboração do manuscrito: RMSMD. Revisão do manuscrito e aprovação da versão final: RMSMD, VS, MCL.

Conflito de Interesses: Os autores declaram não haver conflito de interesses. 